\title{
MicroRNA-145 sensitizes cervical cancer cells to low-dose irradiation by downregulating OCT4 expression
}

\author{
SIQI YAN ${ }^{1}$, XIANGJUN LI ${ }^{2}$, QIAO JIN ${ }^{3}$ and JUN YUAN ${ }^{1}$ \\ ${ }^{1}$ Department of Oncology, Xiangya Hospital, Central South University, Changsha, Hunan 410008; ${ }^{2}$ Department of Oncology, \\ The Second People's Hospital, Changsha, Hunan 410000; ${ }^{3}$ Department of Oncology, Third Xiangya Hospital, \\ Central South University, Changsha, Hunan 410013, P.R. China
}

Received June 26, 2015; Accepted August 23, 2016

DOI: $10.3892 / \mathrm{etm} .2016 .3731$

\begin{abstract}
Poor elucidation of the mechanisms involved in regulating the radiosensitivity of cancers prevents the extensive application of low-dose radiotherapy in clinical settings. The present study was conducted to investigate the role of microRNA-145 (miR-145) in the modulation of cervical cancer cell radiosensitivity, as well as to identify the underlying target of miR-145 during this process. Cervical cancer tera cells were initially exposed to doses of radiation between 1 and 6 Gy before the assessments of the cell viability and apoptosis rate. Irradiation at dose of $1 \mathrm{~Gy}$ was screened as optimum dose and used in subsequent experiments. A dual luciferase reporter assay was performed to demonstrate that octamer-binding transcription factor 4 (OCT4) is a target of miR-145 in cervical cancer. Consequently, OCT4 was suggested to be a target of miR-145, as a dual luciferase vector that was ligated to a fragment corresponding to the predicted target site of miR-145 in OCT4 3'-UTR showed an $83 \%$ reduction in fluorescence. Following exposure to $1 \mathrm{~Gy}$ irradiation, tera cells transfected with miR-145 mimics, which showed downregulation of OCT4 and cyclin D1, had lower cell viability and cell migration rate and higher apoptosis rate compared to non-transfected cells. However, the co-transfection of miR-145 mimics and OCT4 expression vector restored OCT4 and cyclin D1 expression levels and made no significant difference in terms of cell viability, cell migration rate and apoptosis rate. The present results indicate that miR-145 increases the radiosensitivity of cervical cancer cells by silencing OCT4, that cyclin D1 is putatively under the positive regulation of OCT4 and mediates miR-145 function.
\end{abstract}

Correspondence to: Professor Jun Yuan, Department of Oncology, Xiangya Hospital, Central South University, 87 Xiang Ya Road, Changsha, Hunan 410008, P.R. China

E-mail:mtwmdh@163.com

Key words: microRNA-145, cervical cancer, low-dose irradiation, octamer-binding transcription factor 4 , radiosensitivity

\section{Introduction}

Cervical cancer is highly common in women worldwide, with an estimated global incidence of 470,000 new cases each year (1). Currently, radiotherapy remains the most effective therapeutic method for cervical cancer, particularly for the patients at an advanced stage, and can achieve relatively satisfactory outcome in clinical practice (2). However, a range of side effects are associated with conventional high-dose radiotherapy, and thus numerous patients ultimately discontinue radiotherapy due to the severe discomfort involved $(3,4)$. By contrast, low-dose radiotherapy exhibits the advantages of reduced collateral damage, increased safety and easier acceptance by patients, and may therefore offer a promising approach in the field of radiotherapy (5). However, it remains unclear how to guarantee a satisfactory therapeutic effect when diminishing the dose of irradiation (6). Sensitizing cervical cancer cells to irradiation has been proved to be a viable approach, mainly based on recent molecular biotechnology that can modulate corresponding genes to the end of promoting cancer cell radiosensitivity (7).

MicroRNAs (miRNAs) are a class of non-coding RNAs that regulate protein expression by inducing mRNA degradation or interfering with translation, and have been shown to play important role in cancer suppression or carcinogenesis (8). miR-145 has been verified to be important in cancer suppression (9). Downregulation of miR-145 has been widely observed in cervical cancer and several other cancer types $(9,10)$. Artificially promoting the expression of miR-145 by plasmid transfection shows obvious growth inhibition on cancer cells $(11,12)$. However, few studies have been published documenting the role of miR-145 in modulating the radiosensitivity of cancers.

Octamer-binding transcription factor 4 (OCT4) is a stem-related transcription factor, a group of proteins that was initially identified as being involved in the self-renewal and differentiation of embryonic stem cells. However, a large number of clinical reports suggesting that higher expression levels of OCT4 may be associated with higher grades of cancer suggest the function of OCT4 in cancers (13-15). Subsequent research has shown that OCT4 expression in oral cancer is positively correlated with cancer cisplatin resistance, invasion and proliferation (16). OCT4 downregulation via RNA interference 
in head neck squamous cell carcinoma causes an increase in radiosensitivity and a loss of metastatic potential (17). These result further indicate the importance of OCT4 contributing to the development of cancers in a number of ways $(18,19)$.

The aim of the present study was to investigate the role of miR-145 in the modulation of cervical cancer cell radiosensitivity, using transfection with miR-145 mimics to upregulate miR-145 in cervical cancer tera cells. Furthermore, we investigated cell viability, apoptosis rate and migration rate after these cells were exposed to low-dose irradiation. Our study and others' (20) have indicated that OCT4 is an important target of miR-145. Thus, in the present study cervical cancer tera cells in the round were co-transfected with miR-145 mimics and OCT4 expression vector to determine whether OCT4 mediated miR-145 function. This study aimed to provide a theoretical foundation for the modulation of the radiosensitivity of cervical cancer tera cells during low-dose radiotherapy.

\section{Materials and methods}

Cell lines. The cervical cancer Tera cell line was purchased from the American Type Culture Collection (Manassas, VA, USA) and were maintained as exponentially growing monolayers in Dulbecco's modified Eagle's medium (Gibco; Thermo Fisher Scientific, Inc., Grand Island, NY, USA) containing 10\% fetal bovine serum (HyClone; GE Healthcare Life Sciences, Chalfont, UK) in a $37^{\circ} \mathrm{C}$ incubator with $5 \% \mathrm{CO}_{2}$.

Irradiation. Tera cells were trypsinized (Invitrogen; Thermo Fisher Scientific, Inc., Waltham, MA, USA) and seeded into six-well plates with $\sim 1.0 \times 10^{5}$ cells per well. After $48 \mathrm{~h}$ of incubation, the cells were cultured in the medium without fetal bovine serum and irradiated at dose of 1, 2, 4 or 6 Gy on ice. Based on our evaluation of cell viability and apoptosis rate after irradiation, 1 Gy irradiation was selected and performed in following formal test.

Cell survival ratio assay. A Cell Counting Kit-8 (CCK-8; Sigma-Aldrich; Merck KGaA, Darmstadt, Germany) assay was used to measure cell survival ratio after irradiation. $0.5 \times 10^{4}$ cells were seeded in each 96 -well plate for $24 \mathrm{~h}$, and incubated with CCK-8 reagents at a final concentration of $10 \%$ for $1 \mathrm{~h}$. The optical density in each well was determined using an enzyme immunoassay analyzer at $490 \mathrm{~nm}$.

Flow cytometry method. Apoptosis ratio after irradiation was analyzed in vitro using a FACS Annexin V assay kit (BD Biosciences, San Jose, CA, USA) according to the manufacturer's instructions. Briefly, the harvested cells were washed and resuspended in $0.1 \mathrm{M}$ phosphate-buffered saline (PBS). Next, cells were fixed overnight with $75 \%$ cold ethanol, washed twice with cold PBS, then incubated in PBS buffer containing $50 \mu \mathrm{g} / \mathrm{ml}$ propidium iodide (PI) and $20 \mu \mathrm{g} / \mathrm{ml}$ RNase A for $30 \mathrm{~min}$ at $37^{\circ} \mathrm{C}$. Next, cells were incubated with $5 \mu 1$ Annexin $\mathrm{V}$-FITC in $195 \mu \mathrm{l}$ binding buffer in the dark for $10 \mathrm{~min}$. PI and forward light scattering were detected using a FACSCalibur flow cytometer (BD Biosciences) equipped with the ModFit LT software package (version 3.2; Verity Software House, Inc., Topsham, ME, USA).
Dual luciferase reporter assay. Dual luciferase vector pRL-TK was purchased from Promega Corporation (Madison, WI, USA. An oligonucleotide duplex containing the predicted binding site of miR-145 (miRNA response element; MRE) present in the 3'-UTR of OCT4 was inserted into pRL-TK to construct an miR-145 MRE luciferase reporter (pRL-TK-OCT4 3'-UTR). This reporter and negative control were then transfected into tera cells using Lipofectamine 2000 (Invitrogen; Thermo Fisher Scientific, Inc.), according to the manufacturer's recommendations. Firefly luciferase and Renilla reniformis signals were measured $48 \mathrm{~h}$ after transfection using GloMax 20/20n luminometer (Promega Corporation).

Transfection treatment. Overexpression of miR-145 in tera cells was achieved by transfection with miR-145 mimics (GenePharma, Co., Ltd, Shanghai, China) using Lipofectamine 2000 according to the manufacturer's instructions. OCT4 expression vector, the full-length OCT4-coding sequence was amplified and cloned into a pEGFP-C1 expression vector (Invitrogen). Co-transfection of miR-145 mimics and OCT4 expression vector into tera cells was performed using Lipofectamine 2000. Total RNA and protein were extracted from tera cells for subsequent polymerase chain reaction (PCR) and western blot analyses for detecting the mRNA and protein expression levels of miR-145 and OCT4.

Reverse transcription-quantitative PCR (RT-qPCR). TRIzol reagent (Invitrogen) was used to extract total RNA from tera cells. Reversing transcribed RNA $(1 \mu \mathrm{g})$ into cDNA was performed using a MiScript Reverse Transcription Kit (Bio-Rad Laboratories, Inc., Hercules, CA, USA) according to the manufacturer's instructions. Gene expression of miR-145 was assessed using a Power SYBR ${ }^{\circledR}$ Green PCR Master Mix (Applied Biosystems; Thermo Fisher Scientific, Inc.). The following amplification parameters were used: $95^{\circ} \mathrm{C}$ for $10 \mathrm{~min}$, followed by 50 cycles of $95^{\circ} \mathrm{C}$ for $15 \mathrm{sec}, 60^{\circ} \mathrm{C}$ for $1 \mathrm{~min}$, and $95^{\circ} \mathrm{C}$ for $15 \mathrm{sec}$. The following primers were used: miR-145, forward 5'-GTCCTCACGGTCCAGTTT-3' and reverse 5'-TTTGGCACTAGCACATT-3'; U6, forward 5'-CTC GCTTCGGCAGCACA-3' and reverse 5'-AACGCTTCACGA ATTTGCGT-3'. The assay was repeated three times, and gene expression levels were normalized against U6, and calculated using the $2^{-\Delta \mathrm{Ct}}$ method (21). Replacing RNA or cDNA with equal quantities of deionized water was used as the negative control.

Western blot analysis. Cells were lysed on ice in lysis buffer (50 mM Tris- $\mathrm{HCl}$, pH 7.4; $150 \mathrm{mM} \mathrm{NaCl} ; 2$ mM EDTA; $1 \%$ NP-40; and $0.1 \%$ SDS). A total of $20 \mu \mathrm{g}$ protein extracted from cell lysis was separated using 10\% SDS-PAGE and transferred onto a nitrocellulose membrane (Merck Millipore, Billerica, MA, USA). The membrane was then blocked with $5 \%$ bovine serum albumin (Santa Cruz Biotechnology, Inc., Dallas, TX, USA) at room temperature for $1 \mathrm{~h}$, and incubated with the following primary murine monoclonal antibodies at $4^{\circ} \mathrm{C}$ overnight: Anti-OCT4 (1:500; sc-9081; Santa Cruz Biotechnology, Inc.), anti-cyclin D1 (1:500; \#2926; Cell Signaling Technology, Inc., Danvers, MA, USA) and anti- $\beta$-actin (1:500; sc-47778; Santa Cruz Biotechnology, Inc.). In the following steps, membrane underwent at least three washes with 0.1 M PBST 

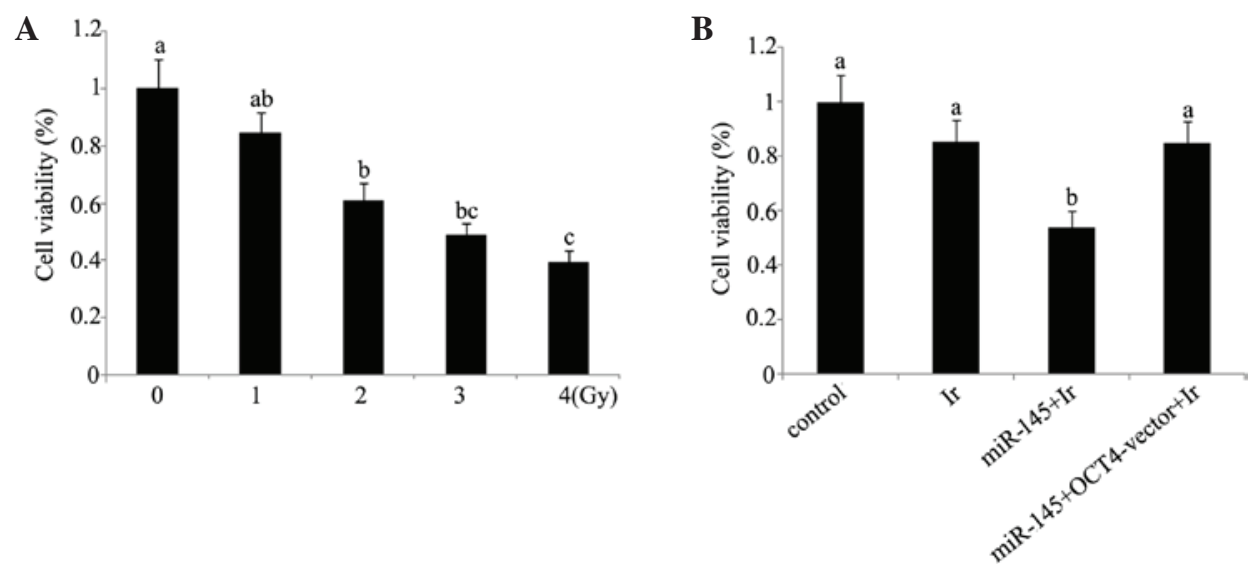

Figure 1. Cell viability of cervical cancer tera cells. (A) Viability of tera cells after exposure to $0,1,2,3$ or 4 Gy of irradiation. (B) Before exposure to 1 Gy irradiation, tera cells were transfected with miR-145 or co-transfected with miR-145 and OCT4 expression vector. The experiment was repeated at least three times. Each bar represents the mean of three independent experiments. Bars not sharing a common letter differ $(\mathrm{P}<0.05)$. Ir, irradiation at dose of $1 \mathrm{~Gy}$; miR-145, microRNA-145 mimics; OCT4-vector, octamer-binding transcription factor 4 expression vector.

before incubation with horseradish peroxidase-conjugated secondary antibodies (1:10,000; sc-2004 and sc-2005; Santa Cruz Biotechnology, Inc.) for $2 \mathrm{~h}$ at room temperature. Bands were detected using an enhanced chemiluminesence detection kit (Pierce Protein Biology; Thermo Fisher Scientific, Inc.). Relative quantification was determined with the AlphaView system (version 3.4.0.729; ProteinSimple, Santa Clara, CA, USA), using $\beta$-actin as the loading control.

Wound healing assay. Cells were trypsinized and seeded in equal numbers $\left(1 \times 10^{5}\right.$ cells/well) into six-well tissue culture plates, and allowed to grow to confluence $(85 \% ; \sim 24 \mathrm{~h})$. A $100-\mu 1$ pipette tip was used to create an artificial wound by scratching a homogenous line on the cell monolayer. After scratching, the cells were washed and cultured in serum-free medium. The microscopic images of same area were collected immediately after a wound was inflicted to the cell and at time point $24 \mathrm{~h}$. Migration rates were calculated using the following equation: (Initial distance - final distance / initial distance) x 100.

Statistical analysis. SPSS statistical software, version 19.0 (IBM SPSS, Armonk, NY, USA) was used for statistical analysis. One-way analysis of variance with post-hoc $t$-testing was used for multiple comparisons between each group. Data are expressed as the mean \pm standard deviation. $\mathrm{P}<0.05$ was considered to indicate a statistically significant difference.

\section{Results}

Cell viability and apoptosis analyses indicate that 1 Gy is an appropriate dose for modelling low-dose radiotherapy in vitro. In our preliminary experiment, cervical cancer tera cells were exposed to 1,2, 4 and 6 Gy of irradiation. Based on the evaluation of cell viability (Fig. 1) and apoptosis rate (Fig. 2) after irradiation (Figs. 1A and 2A), irradiation-induced cell damage was increased with the elevation of the irradiation dose and reached the most severe level at 6 Gy irradiation, with $\sim 50 \%$ cell viability and $45 \%$ apoptosis rate of the control cells, while there was no significant damage inflicted by 1 Gy irradiation.
All subsequent experiments were performed using irradiation at dose of $1 \mathrm{~Gy}$, as this dosage was an appropriate model of low-dose radiotherapy for enhancing the radiosensitivity.

$m R N A$ and protein expression levels. In dual luciferase reporter assay, a dual luciferase vector that was ligated to a fragment corresponding to the predicted target site of miR-145 in OCT4 3'-UTR reduced by $83 \%$ fluorescence (Fig. 3A). Furthermore, RT-qPCR analysis showed that miR-145 was significantly upregulated in tera cells transfected with miR-145 mimics or co-transfected with miR-145 mimics and OCT4 expression vector before cell exposure to $1 \mathrm{~Gy}$ irradiation (Fig. 3B). Exposure to $1 \mathrm{~Gy}$ irradiation resulted in the signifieant reduction of cyclin D1 protein expression $(\mathrm{P}<0.05)$, but not of OCT4 protein expression (Fig. 4). After irradiation, tera cells that were initially transfected with miR-145 mimics showed marked inhibition of their protein expression levels of OCT4 and cyclin D1 compared with those in non-treated tera cells. However, this inhibition was not observed in tera cells co-transfected with miR-145 mimics and OCT4 expression vector.

Cell viability and apoptosis rate. Tera cells transfected with miR-145 mimics exhibited a significant reduction in post-irradiation cell viability and increase of post-irradiation apoptosis rate $(\mathrm{P}<0.05)$. By contrast, similar reductions were not observed in tera cells co-transfected with miR-145 mimics and OCT4 expression vector (Figs. 1B and 2B). In the would healing assay (Fig. 5), the cell migration rate exhibited a significant reduction following cell exposure to $1 \mathrm{~Gy}$ irradiation $(\mathrm{P}<0.05)$. Transfection with miR-145 mimics before irradiation rendered cell migration rate further attenuated $(\mathrm{P}<0.05$ vs. cell exposure to irradiation only; $\mathrm{P}<0.01$ vs. control). Co-transfection with miR-145 mimics and OCT4 expression vector before irradiation restored cell migration rate close to that of cell exposure to irradiation only ( $\mathrm{P}<0.05$ vs. control).

\section{Discussion}

miRNAs are characterized by negatively regulating the expression levels of numerous key proteins involved in 
A
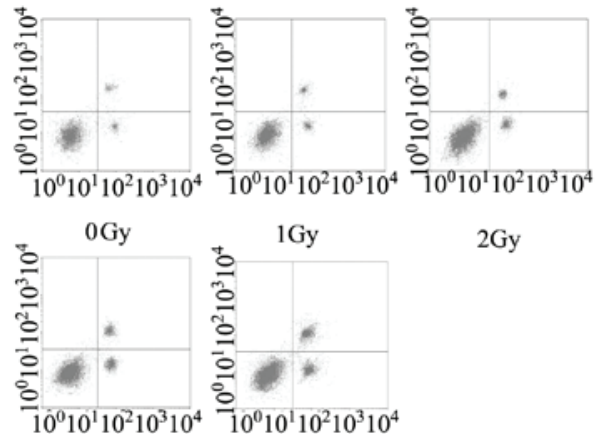

4 Gy

$\mathbf{B}$
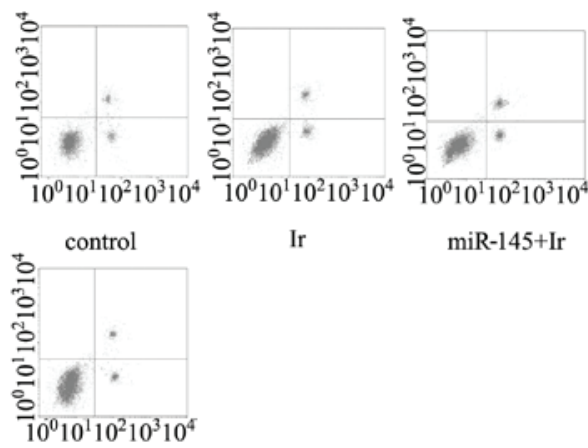

Ir

miR-145+Ir

2Gy
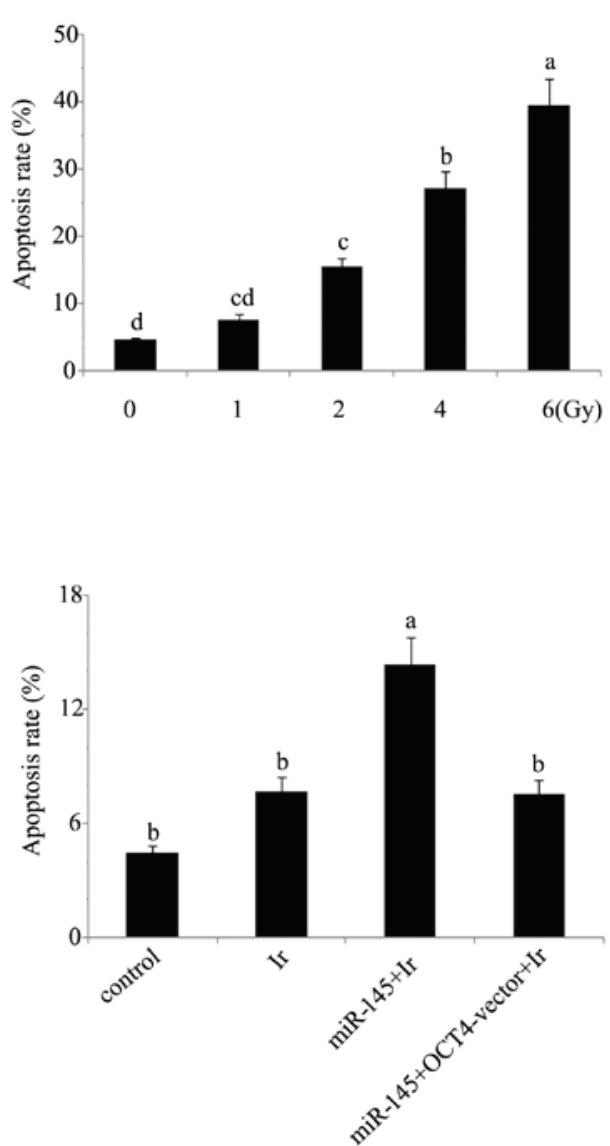

Figure 2. Apoptosis rates of cervical tera cells were tested using Annexin V-fluorescein isothiocyanate/propidium iodide apoptosis kit and flow cytometry. (A) Apoptosis rate of tera cells after exposure to different dose of irradiation. (B) Before exposure to 1 Gy irradiation, tera cells were transfected with miR-145 mimics or co-transfected with miR-145 and OCT4 expression vector. The horizontal axes of the flow cytometer images represent Annexin V-fluorescein isothiocyanate, and the vertical axes represent propidium iodide. The experiment was repeated at least three times. Each bar represents the mean of three independent experiments. Bars not sharing a common letter differ $(\mathrm{P}<0.05)$. Ir, irradiation at dose of 1 Gy; miR-145, microRNA-145 mimics; OCT4-vector, octamer-binding transcription factor 4 expression vector.
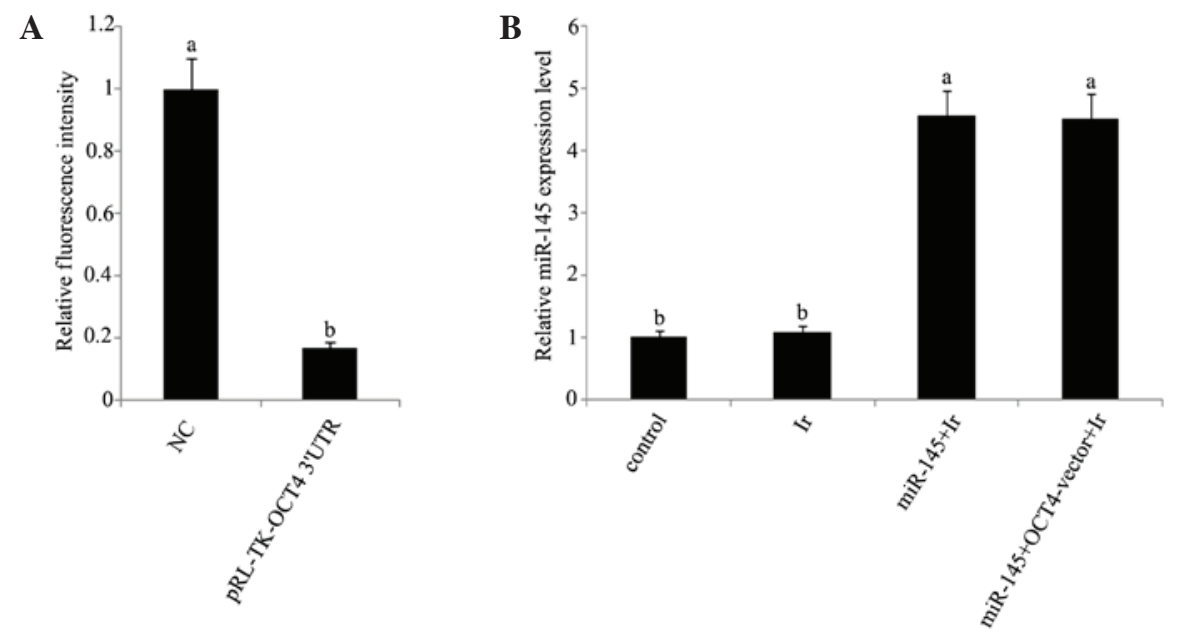

Figure 3. Dual luciferase reporter assay and quantitative polymerase chain reaction analysis of miR-145 expression in cervical tera cells after different treatments. (A) Dual luciferase reporter ligated to putative miR-145 binding sequences in the OCT4 3'-UTR (pRL-TK-OCT4 3'-UTR) and NC were transfected into tera cells. (B) Before exposure to $1 \mathrm{~Gy}$ irradiation, tera cells were transfected with miR-145 mimics or co-transfected with miR-145 and OCT4 expression vector. The experiment was repeated at least three times. Each bar represents the mean of three independent experiments. Bars not sharing a common letter differ $(\mathrm{P}<0.05)$. NC, normal control; Ir, irradiation at dose of $1 \mathrm{~Gy}$; miR-145, microRNA-145 mimics; OCT4-vector, octamer-binding transcription factor 4 expression vector.

psychological and pathological processes, and have been associated with regulating various hallmarks of cancer $(9,10)$. However, there uncertainty remains regarding the functional effects of each miRNA in specific cancer types. The present results suggest that miR-145 promotes the radiosensitivity of cervical cancer tera cells, as demonstrated by the finding that 

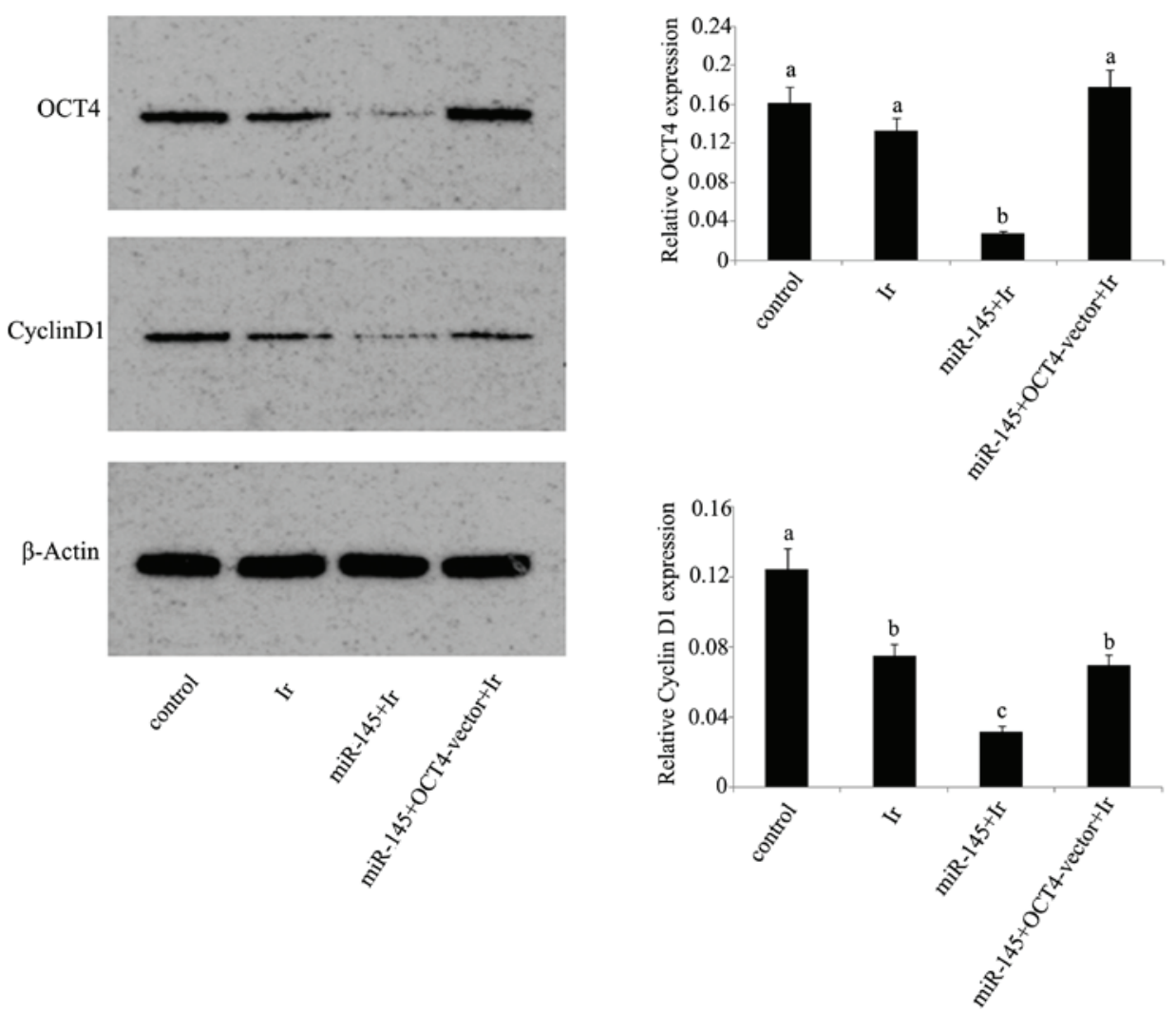

Figure 4. Protein expression levels of OCT4 and cyclin D1 in cervical tera cells after different treatments. Before exposure to 1 Gy irradiation, tera cells were transfected with miR-145 mimics or co-transfected with miR-145 and OCT4 expression vector. The relative protein expression levels of OCT4 and cyclin D1 in tera cells after different treatments were tested by western blot and normalized against $\beta$-actin. The experiment was repeated at least three times. Each bar represents the mean of three independent experiments. Bars not sharing a common letter differ $(\mathrm{P}<0.05)$. Ir, irradiation at dose of $1 \mathrm{~Gy}$; miR-145, microRNA-145 mimics; OCT4-vector, octamer-binding transcription factor 4 expression vector.

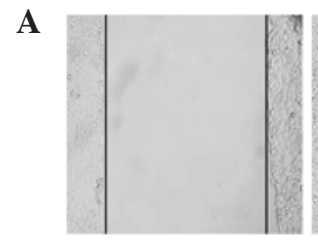

$0 \mathrm{~h}$

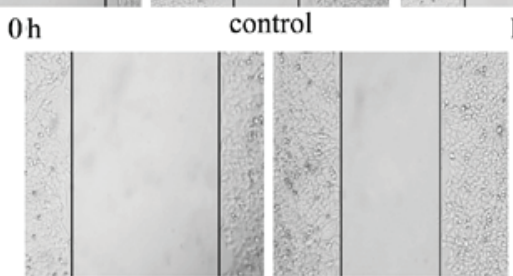

miR-145+Ir miR-145+OCT4-vector+Ir

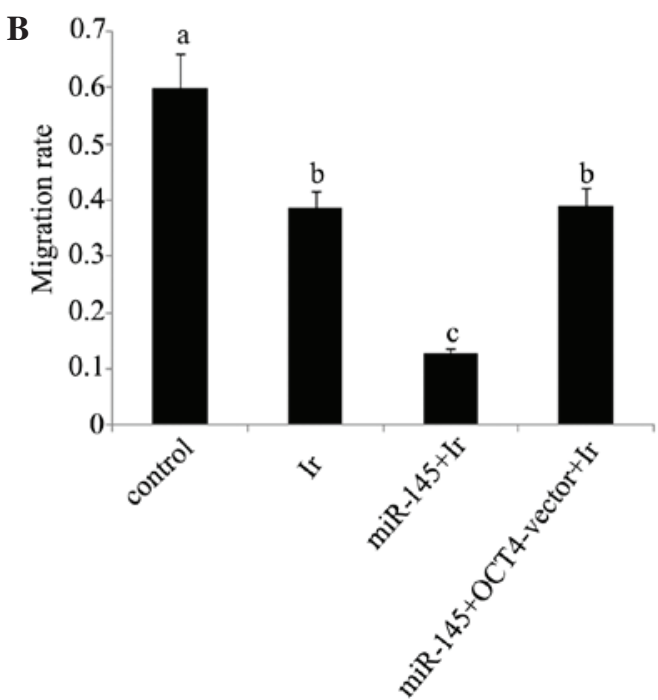

Figure 5. Cell migration rate of cervical tera cells. (A) Cell migration rate of tera cells after exposure to different dose of irradiation was tested by using wound healing test. (B) Before exposure to $1 \mathrm{~Gy}$ irradiation, tera cells were transfected with miR-145 mimics or co-transfected with miR-145 and OCT4 expression vector. All experiments were repeated at least three times. Each bar represents the mean of three independent experiments. Bars not sharing a common letter differ $(\mathrm{P}<0.05)$. Ir, irradiation at dose of $1 \mathrm{~Gy}$; miR-145, microRNA-145 mimics; OCT4-vector, octamer-binding transcription factor 4 expression vector.

miR-145 overexpression via the transfection with miR-145 mimics significantly decreased post-irradiation cell viability of tera cells and enhanced its post-irradiation apoptosis rate.

miR-145 is documented to be suppressive to cell growth of cancer cells $(9,10)$. It has been revealed that miR-145 overexpression correlates with breast cancer MCF-7 cell growth inhibition (11). The loss of miR-145 serves as a selective advantage for the growth of colon, cervical and bladder cancers (12). However, the function of miR-145 as a cancer growth inhibitor does not necessarily mean that miR-145 
can enhance the radiosensitivity of cancers, which involves numerous mechanisms responsible for death-inducing effects after radiation damage (22). It has been demonstrated that exposure to radiation may result in the generation of substantial oxidative free radicals which have harmful effects on DNA via deteriorating its original molecule structures (23). DNA injury is a strongly positive signal for the initiation of apoptosis, and cells that are less sensitive to radiation are observed to evade cell death predominantly by blocking and interfering apoptosis signals and/or immediately repairing injured DNA (4). The present results showed that tera cells with elevated miR-145 had much lower cell viability and a higher apoptosis rate after cell exposure to low-dose irradiation, indicating that miR-145 enhances the sensitivity of cervical cancer cells to radiation.

The present study, and prior experiments (20), showed that the endogenous OCT4 protein level was significantly downregulated in tera cells transfected with miR-145 mimics, suggesting that OCT4 expression is negatively regulated by miR-145 in tera cells. Further experiments involving the co-transfection of tera cells with miR-145 mimics and an OCT4 expression vector, to remove the inhibitory effect of miR-145 on OCT4 expression, showed that miR-145 significantly decreased post-irradiation cell viability and that the enhanced post-irradiation apoptosis rate was abrogated. These data collectively indicate that miR-145 enhancing radiosensitivity occurs primarily via silencing of OCT4.

Previous results indicate that OCT4 facilitates cell proliferation and inhibits apoptosis. It has been reported that OCT4 promotes the proliferation of esophageal squamous cell carcinoma by positively regulating the expression of survivin, which is an important member of the inhibitors of the apoptotic gene family (24). Furthermore, OCT4 has been shown to influence survival signal pathways, including those mediated by Tcl1/Akt1, signal transducer and activator of transcription 3 and tumor protein p53 in various cancer types (25-27). Previous data suggest that OCT4 directly induces expression of miR-125b, which inhibits its target, Bcl-2 antagonist/killer 1, leading to the suppression of cervical cancer cell apoptosis (28). OCT4 harboring anti-apoptosis property may to some extent takes the responsibility that OCT4 attenuates the radiosensitivity.

Cyclin D1 is also a key mediator that contributes to reduce cancer cell radiosensitivity via an established mechanism that facilitates G1-S cell cycle transition to improve cell self-renew and proliferation after irradiation (29-31). In the present study, cyclin D1 downregulation was observed in cells exposed to irradiation and cells transfected with miR-145 mimics was associated with considerable reduction of cell migration rate in wound healing assay. Wound healing assays may be used to detect cellular self-repairing capability following wound. A lower cell migration rate indicates slower proliferation and weaker self-repairing capability, which in turn suggests a higher radiosensitivity (32). Furthermore, previous studies have reported that upregulated cyclin D1 was associated a high incidence of cervical lymph node metastasis of squamous cell carcinoma (33). In addition, prior experiments suggest that cyclin D1 serves a crucial function in processes leading to an increase in metastatic potential, such as migratory and invasive properties, potentially through increasing matrix metalloproteinase activity and cellular motility $(34,35)$. It is widely accepted that cancer metastasis facilitates cancer cells to evade irradiation (36). Thus, the present observation of downregulated cyclin D1 and lower cell migration rate indicates potential utility in clinical radiotherapy. However, there is limited evidence that cyclin D1 is directly regulated by miR-145. As cyclin D1 exhibited similar variations in protein expression with OCT4 in the present test, cyclin D1 was hypothesized to be under the positive regulation of OCT4. Contradictorily, previous results suggest that cyclin D1 is negatively regulated by OCT4 in human embryonic stem cells (37). This difference may be due to the different types of cells used in each experiment, or it is possible that cyclin D1 is under more complicated regulation than our hypothesized mechanism.

In summary, a dual luciferase reporter assay verified that OCT4 is an important target of miR-145 in cervical cancer tera cells. Transfection with miR-145 mimics repressed OCT4 expression and promoted radiosensitivity of cervical cancer tera cells. However, co-transfection of miR-145 mimic and OCT4 expression vector removed the inhibition of miR-145 to OCT4 and abrogated the enhancement of miR-145 to radiosensitivity, suggesting that the miR-145-associated increase in the radiosensitivity of cervical cancer cells is a result of OCT4 silencing. In addition, cyclin D1 was inhibited by miR-145, but co-transfection with miR-145 mimics and OCT4 expression vector that restored OCT4 expression and led to the recovery of cyclin D1 expression. Thus, it is speculated that cyclin D1 is under the positive regulation of OCT4. However, this concept is contradictory to previous research (37). Further research investigating the mechanism by which cyclin D1 is regulated by miR-145 and/or OCT4 are required.

\section{Acknowledgements}

The authors thank Professor Jun Yuan for his valuable suggestions and critical reading of the manuscript.

\section{References}

1. Wang X, Tang S, Le SY, Lu R, Rader JS, Meyers C and Zheng ZM: Aberrant expression of oncogenic and tumor-suppressive microRNAs in cervical cancer is required for cancer cell growth. PLoS One 3: 2557, 2008.

2. Mongula J, Slangen B, Lambregts D, Bakers F, Mahesh S, Lutgens L, Van Gorp T, Vliegen R, Kruitwagen R and Beets-Tan R: Predictive criteria for MRI-based evaluation of response both during and after radiotherapy for cervical cancer. J Contemp Brachytherapy 8: 181-188, 2016.

3. Kitahara O, Katagiri T, Tsunoda T, Harima Y and Nakamura Y: Classification of sensitivity or resistance of cervical cancers to ionizing radiation according to expression profiles of 62 genes selected by cDNA microarray analysis. Neoplasia 4: 295-303, 2002.

4. Liu SS, Chan KY, Leung RC, Law HK, Leung TW and Ngan HY: Enhancement of the radiosensitivity of cervical cancer cells by overexpressing p73alpha. Mol Cancer Ther 5: 1209-1215, 2006.

5. Liu R, Wang X, Tian JH, Yang K, Wang J, Jiang L and Hao XY: High dose rate versus low dose rate intracavity brachytherapy for locally advanced uterine cervix cancer. Cochrane Database Syst Rev 9: CD007563, 2014.

6. Hareyama M, Sakata K, Oouchi A, Nagakura H, Shido M, Someya M and Koito K: High-dose-rate versus low-dose-rate intracavitary therapy for carcinoma of the uterine cervix: A randomized trial. Cancer 94: 117-124, 2002.

7. Cui H, Qin Q, Yang M, Zhang H, Liu Z, Yang Y, Chen X, Zhu H, Wang D, Meng C, et al: Bortezomib enhances the radiosensitivity of hypoxic cervical cancer cells by inhibiting HIF-1 $\alpha$ expression. Int J Clin Exp Pathol 8: 9032-9041, 2015. 
8. Reshmi G and Pillai MR: Beyond HPV: Oncomirs as new players in cervical cancer. FEBS Lett 582: 4113-4116, 2008.

9. Xue M,Zhao L, Yang F, Li Z and Li G: MicroRNA-145 inhibits the malignant phenotypes of gastric carcinoma cells via downregulation of fascin 1 expression. Mol Med Rep 13: 1033-1039, 2016.

10. Han T, Yi XP,Liu B, Ke MJ and Li YX: MicroRNA-145 suppresses cell proliferation, invasion and migration in pancreatic cancer cells by targeting NEDD9. Mol Med Rep 11: 4115-4120, 2015.

11. Wang S, Bian C, Yang Z, Bo Y, Li J, Zeng L, Zhou H and Zhao RC: miR-145 inhibits breast cancer cell growth through RTKN. Int J Oncol 34: 1461-1466, 2009.

12. Ostenfeld MS, Bramsen JB, Lamy P, Villadsen SB, Fristrup N, Sørensen KD, Ulhøi B, Borre M, Kjems J, Dyrskjøt L and Orntoft TF: miR-145 induces caspase-dependent and -independent cell death in urothelial cancer cell lines with targeting of an expression signature present in Ta bladder tumors. Oncogene 29: 1073-1084, 2010.

13. Gu TT, Liu SY and Zheng PS: Cytoplasmic NANOG-positive stromal cells promote human cervical cancer progression. Am J Pathol 181: 652-661, 2012.

14. Vaiphei K, Sinha SK and Kochhar R: Comparative analysis of Oct4 in different histological subtypes of esophageal squamous cell carcinomas in different clinical conditions. Asian Pac J Cancer Prev 15: 3519-3524, 2014

15. Vargas TH, Pulz LH, Barra CN, Kleeb SR, Xavier JG Catão-Dias JL, Fukumasu H, Nishiya AT, Strefezzi RF: Immunohistochemical expression of the pluripotency factor OCT4 in canine mast cell tumours. J Comp Pathol. 153: 251-255, 2015.

16. Tsai LL, Yu CC, Chang YC, Yu CH and Chou MY: Markedly increased Oct4 and Nanog expression correlates with cisplatin resistance in oral squamous cell carcinoma. J Oral Pathol Med 40: 621-628, 2011.

17. Lo WL, Chien Y, Chiou GY, Tseng LM, Hsu HS, Chang YL, $\mathrm{Lu} \mathrm{KH}$, Chien CS, Wang ML, Chen YW, et al: Nuclear localization signal-enhanced RNA interference of EZH2 and Oct4 in the eradication of head and neck squamous cell carcinoma-derived cancer stem cells. Biomaterials 33: 3693-3709, 2012.

18. Kuo KK, Lee KT, Chen KK, Yang YH, Lin YC, Tsai MH, Wuputra K, Lee YL, Ku CC, Miyoshi H, et al: Positive feedback loop of OCT4 and c-JUN expedites cancer stemness in liver cancer. Stem Cells: Jun 24, 2016 (Epub ahead of print).

19. Lu CS, Shieh GS, Wang CT, Su BH, Su YC, Chen YC, Su WC, Wu P, Yang WH, Shiau AL and Wu CL: Chemotherapeutics-induced Oct 4 expression contributes to drug resistance and tumor recurrence in bladder cancer. Oncotarget: May 26 , 2016 (Epub ahead of print).

20. Xu N, Papagiannakopoulos T, Pan G, Thomson JA and Kosik KS: MicroRNA-145 regulates OCT4, SOX2 and KLF4 and represses pluripotency in human embryonic stem cells. Cell 137: 647-658, 2009.

21. Livak KJ and Schmittgen TD: Analysis of relative gene expression data using real-time quantitative PCR and the $2^{-\Delta \Delta C}$ method. Methods 25: 402-408, 2001.

22. Dote H, Burgan WE, Camphausen K and Tofilon PJ: Inhibition of hsp90 compromises the DNA damage response to radiation. Cancer Res 66: 9211-9220, 2006.

23. Shuryak I and Brenner DJ: A model of interactions between radiation-induced oxidative stress, protein and DNA damage in Deinococcus radiodurans. J Theor Biol 261: 305-317, 2009.
24. Li C, Yan Y, Ji W, Bao L, Qian H, Chen L, Wu M, Chen H, $\mathrm{Li} \mathrm{Z}$ and Su C: OCT4 positively regulates Survivin expression to promote cancer cell proliferation and leads to poor prognosis in esophageal squamous cell carcinoma. PLoS One 7: e49693, 2012.

25. Hu T, Liu S, Breiter DR, Wang F, Tang Y and Sun S: Octamer 4 small interfering RNA results in cancer stem cell-like cell apoptosis. Cancer Res 68: 6533-6540, 2008.

26. Lin Y, Yang Y, Li W, Chen Q, Li J, Pan X, Zhou L, Liu C, Chen $\mathrm{C}$, aself-renewal and survival of embryonal carcinoma cells. Mol Cell 48: 627-640, 2012.

27. Zhang Z, Zhu Y, Lai Y, Wu X, Feng Z, Yu Y, Bast RC Jr, Wan X, Xi X and Feng Y: Follicle-stimulating hormone inhibits apoptosis in ovarian cancer cells by regulating the OCT4 stem cell signaling pathway. Int J Oncol 43: 1194-1204, 2013.

28. Wang YD, Cai N, Wu XL, Cao HZ, Xie LL and Zheng PS: OCT4 promotes tumorigenesis and inhibits apoptosis of cervical cancer cells by miR-125b/BAK1 pathway. Cell Death Dis 4: e760, 2013.

29. Jeselsohn R, Brown NE, Arendt L, Klebba I, Hu MG, Kuperwasser $\mathrm{C}$ and Hinds PW: Cyclin D1 kinase activity is required for the self-renewal of mammary stem and progenitor cells that are targets of MMTV-ErbB2 tumorigenesis. Cancer Cell 17: 65-76, 2010.

30. Shimura T, Noma N, Oikawa T, Ochiai Y, Kakuda S, Kuwahara Y, Takai Y, Takahashi A and Fukumoto M: Activation of the AKT/Cyclin D1/Cdk4 survival signaling pathway in radioresistant cancer stem cells. Oncogenesis 1: e12, 2012.

31. Chu Q, Han N, Yuan X, Nie X, Wu H, Chen Y, Guo M, Yu S and $\mathrm{Wu} \mathrm{K}$ : DACH1 inhibits Cyclin D1 expression, cellular proliferation and tumor growth of renal cancer cells. J Hematol Oncol 7: 73, 2014.

32. Jin Q, Li X and Cao P: EphA2 modulates radiosensitive of hepatocellular carcinoma cells via $\mathrm{p} 38 /$ mitogen-activated protein kinase-mediated signal pathways. Kaohsiung J Med Sci 31: 510-517, 2015.

33. Suresh TN, Hemalatha A, Harendra Kumar ML and Azeem Mohiyuddin SM: Evaluation of histomorphological and immunohistochemical parameters as biomarkers of cervical lymph node metastasis in squamous cell carcinoma of oral cavity: A retrospective study. J Oral Maxillofac Pathol 19: 18-24, 2015.

34. Hwang SJ, Lee HW, Kim HR, Song HJ, Lee DH, Lee H, Shin CH, Joung JG, Kim DH, Joo KM and Kim HH: Overexpression of microRNA-95-3p suppresses brain metastasis of lung adenocarcinoma through downregulation of Cyclin D1. Oncotarget 6: 20434-20448, 2015

35. Chen YJ, Lee LY, Chao YK, Chang JT, Lu YC, Li HF, Chiu CC, $\mathrm{Li}$ YC, Li YL, Chiou JF and Cheng AJ: DSG3 facilitates cancer cell growth and invasion through the DSG3-plakoglobin-TCF/LEF-Myc/cyclin D1/MMP signaling pathway. PLoS One 8: e64088, 2013.

36. Atkinson RL, Zhang M, Diagaradjane P, Peddibhotla S, Contreras A, Hilsenbeck SG, Woodward WA, Krishnan S, Chang JC and Rosen JM: Thermal enhancement with optically activated gold nanoshells sensitizes breast cancer stem cells to radiation therapy. Sci Transl Med 2: 55ra79, 2010.

37. Card DA, Hebbar PB, Li L, Trotter KW, Komatsu Y, Mishina Y and Archer TK: Oct4/Sox2-regulated miR-302 targets Cyclin D1 in human embryonic stem cells. Mol Cell Biol 28: 6426-6438, 2008. 